\title{
Power System Voltage Stability Enhancement Based on Line Voltage Stability Indices in Heavily Loaded and Line Outage Contingency Situations
}

\author{
Sirote Khunkitti ${ }^{1}$, Suttichai Premrudeepreechacharn ${ }^{1}$, Apirat Siritaratiwat ${ }^{2 *}$ and Neville R. Watson ${ }^{3}$ \\ ${ }^{1}$ Department of Electrical Engineering, Faculty of Engineering, Chiang Mai University, Chiang Mai 50200, Thailand, \\ ${ }^{2}$ Department of Electrical Engineering, Faculty of Engineering, Khon Kaen University, Khon Kaen 40002, Thailand, \\ ${ }^{3}$ Department of Electrical and Computer Engineering, University of Canterbury, Christchurch 8140, New Zealand,
}

*Corresponding Author: Apirat Siritaratiwat

ORCID: 0000-0001-8870-7307 (Sirote Khunkitti)

\begin{abstract}
This paper presents power system voltage stability enhancement using line voltage stability indices based on an optimal power flow (OPF) problem in heavily loaded and line outage contingency situations. Voltage stability indices (VSIs) are widespread indicators determining the proximity of a system to voltage collapse, and operators can protect the voltage collapse before it happens. The values of VSIs are generally between 0 (no load condition) and 1 (voltage collapse). By limiting the threshold value of VSIs, operators can move a system to a secure point when the system tends to collapse. Moreover, the values of VSIs can also be minimized in order to improve voltage stability of a system. Therefore, in the OPF problem, the VSIs are proposed as part of the constraint to restrict the threshold of the VSIs and as the objective function to improve the voltage stability. Three line VSIs consisting of Lmn, VCPI and FVSI are compared in terms of transmission line loss reduction and voltage stability improvement in the heavily loaded and line outage contingency situations. Salp swarm algorithm (SSA) is employed to solve the OPF problem. The performance of the VSIs are investigated in the IEEE 30-bus and 118-bus systems. The simulation results show the enhancement of the voltage stability and reduction of loss, especially when using VSIs as the objective function.
\end{abstract}

Keywords: Optimal power flow; Salp swarm algorithm; Voltage stability enhancement; Voltage stability index; Voltage stability constraint; Voltage stability objective

\section{INTRODUCTION}

Due to voltage instability and voltage collapse causing various major blackouts throughout the world [1-4], the necessity to maintain adequate loadability to guarantee steady-state voltage stability in optimal power flow (OPF) problems has led to the enhancement of voltage stability in a power system. Generally, the objective function of a power system in OPF problem is aimed to minimize generation costs so that the minimum expense of power plants is met. Additionally, to ensure system security, voltage magnitude and line flow limits are traditionally considered as the system constraints of the OPF problems. However, in a high risk of voltage instability situations such as heavily loaded and line outage contingency situations, the voltage limit constraint might be insufficient to assure the acceptable voltage stability level, and the objective function should instead mainly focus on the voltage stability enhancement to guarantee the system security. The assessment of how close of a system to voltage collapse is required before the voltage stability enhancement can be done [5].

Many techniques and methods in the literature have been introduced to analyze and predict the voltage collapse proximity [6-8]. Voltage stability index (VSI) is the popular technique adopted to investigate the voltage collapse proximity of a system. There are various VSIs presented in the literature such as L-index employed to indicate the most critical bus [9], line stability index (Lmn) [10], voltage collapse proximity indicator (VCPI) [11], fast voltage stability index (FVSI) [12], LQP index [13] and online voltage stability index (LVSI) [14] where the last five indices were proposed to state the most critical line in the system network.

Normally, VSIs are utilized to forecast the proximity of the system to voltage instability, so the operators can notice it and secure the system from the voltage collapse $[15,16]$. However, VSIs could also be used to enhance the voltage stability of a system by applying as the added constraint or the objective function in the OPF problems. Several works have added VSIs as part of the constraint of the OPF problems; therefore, the value of the VSIs is limited when the system operates close to the instability point and the operators is able to move the system to the secure point $[17,18]$. In addition, VSIs have also been applied as the objective function to be minimized in the OPF problems, so that the value of the VSIs is decreased and the system is more secure from the voltage collapse [19-21].

To solve the OPF problems and obtain optimal setting of the control variables, several techniques have been employed [2225]. However, these techniques are too often trapped in the local optima or appropriate for particular objective function. In the past decades, to deal with these problems, various metaheuristic algorithms such as moth flame algorithm (MSA) [26], particle swarm optimization (PSO) [27], salp swarm algorithm (SSA) 
[28], and hybrid dragonfly algorithm-particle swarm optimization (DA-PSO) [29] have been proposed and successfully solved the OPF problems. SSA is a recent proposed metaheuristic algorithm introduced by Seyedali Mirjalili [28]. It has been applied to problems in many different fields and could efficiently provide better feasible solutions than several algorithms in the literature [30-32]. Thus, SSA is adopted to solve OPF problems in this work.

Studies on the incorporating VSIs as part of the constraint or objective functions in OPF problems have done in different aspects. For example, VCPI was proposed as added constraint to prevent the voltage collapse, and the summation of VCPI of all lines was applied as the objective function to enhance the whole system stability [17]. However, only VCPI was investigated in this work although there are many successfully proposed VSIs in the literature, and only small systems were tested which could not verify the performance of the VSI for large practical systems. In [33], several VSIs were selected as the objective function to compare the effectiveness of the VSIs. This work investigated the enhancement of the voltage stability by observing the maximum loadability before the voltage collapse point; however, the line outage contingency condition where the systems mostly require voltage stability enhancement have not been investigated. In [34], four line VSIs were introduced as the objective function in the line outage contingency condition. The maximum loadability values at the voltage collapse point were also demonstrated. However, the VSIs have not been tested in the heavily loaded condition, and the VSIs were only considered as the objective function even if adding VSIs as part of the constraints is also necessary when system economic and security are required. Hence, the performance comparison of considering several VSIs as the objective function and constraint in the heavily loaded and line outage contingency situations where the operators can effectively choose the appropriate VSIs to enhance the system stability is rarely investigated.

In this paper, various VSIs are considered as the added constraint and the objective function in the OPF problems to improve the voltage stability of a system in heavily loaded and line outage contingency situations. Each case study is investigated on the IEEE 30-bus and 118-bus systems, and the performance of each case is compared with the generation cost objective function referred as a base case. The performance is compared in terms of transmission line loss reduction and voltage stability enhancement. The maximum loadability of each case is also evaluated to observe the ability when systems are enforced to operate close to the voltage collapse point. SSA is employed to solve the OPF problems for all case studies.

\section{LINE VOLTAGE STABILITY INDEX FORMULATIONS}

Line stability indices were introduced to estimate the system voltage stability by detecting the critical transmission line of a system. In this study, three line VSIs are included as provide below.

\section{II.I Lmn}

Line stability index (Lmn) was introduced based on the concept of power flow in a single transmission line presented in Fig 1 [10]. The index formulation is expressed as shown below.

$\mathrm{Lmn}=\frac{4 X Q_{r}}{\left(V_{s} \sin (\theta-\delta)\right)^{2}}$

where $X$ is the line reactance, $Q_{r}$ is the reactive power at the receiving end, $V_{s}$ is the voltage magnitude at the sending end, $\delta$ $=\delta_{s}-\delta_{r}, \delta_{s}$ is the voltage phase angle at the sending end, $\delta_{r}$ is the voltage phase angle at the receiving end, and $\theta$ is the angle of the line impedance.

The value of Lmn is evaluated for all transmission lines and its value is between 0 (no load) and 1 (voltage collapse).

\section{II.II VCPI}

Voltage collapse proximity indicator (VCPI) was proposed to measure system voltage stability [11]. The index was derived from the concept of maximum power transfer in a transmission line as demonstrated in Fig 1. VCPI can be calculated as the provided equations.

$$
\begin{aligned}
& \operatorname{VCPI}(\text { power })=\frac{P_{r}}{P_{r(\max )}} \\
& \operatorname{VCPI}(\text { losses })=\frac{Q_{r}}{Q_{r(\max )}} \\
& P_{r(\max )}=\frac{V_{s}^{2}}{Z} \frac{\cos \phi}{4 \cos ^{2}((\theta-\phi) / 2)} \\
& Q_{r(\max )}=\frac{V_{s}^{2}}{Z} \frac{\sin \phi}{4 \cos ^{2}((\theta-\phi) / 2)}
\end{aligned}
$$

where $P_{r}$ is the active power at the receiving end, $P_{r(\max )}$ is the maximum active power at the receiving end, $Q_{r(\max )}$ is the maximum reactive power at the receiving end, $Z$ is the line impedance and $\phi=\tan ^{-1}\left(Q_{r} / P_{r}\right)$.

VCPI values of all lines are calculated to define the proximity of the system to voltage collapse where the value is in the range of 0 determining no-load condition and 1 determining voltage collapse.

\section{II.III FVSI}

Fast voltage stability index (FVSI) was introduced to investigate the voltage line stability of a system [12]. The FVSI formulation

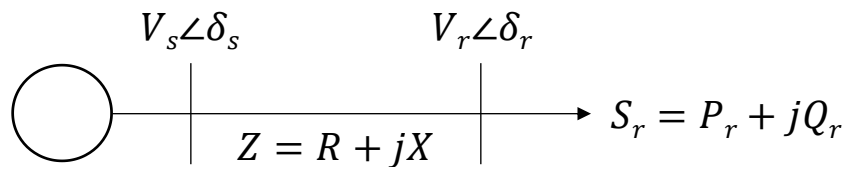

Fig. 1. Single transmission line diagram 
is proposed from the concept of power flow in a transmission line as presented in Fig 1 and can be computed by the following equation.

$$
\mathrm{FVSI}=\frac{4 Z^{2} Q_{r}}{V_{s}^{2} X}
$$

The FVSI is computed for the entire transmission lines, and the condition to indicate the system stability is the same as Lmn and VCPI $(0 \leq$ FVSI $\leq 1)$.

\section{PROBLEM FORMULATIONS}

The goal of the OPF problem is to find the optimal setting of control variables so that optimizing considered objective function while satisfying a set of constraints. The mathematical formulations of the OPF problem can be presented as given below.

$\min f(x, u)$

subject to

$$
\begin{aligned}
& g(x, u)=0 \\
& h(x, u) \leq 0
\end{aligned}
$$

where $x$ is vector of system state/dependent variables, $u$ is vector of control/independent variables, $f(x, u)$ is the objective function to be minimized, $g(x, u)$ is the equality constraints and $h(x, u)$ is the inequality constraints.

\section{III.I Objective functions}

This study focuses on imposing the summation of the line VSIs as the objective function. Traditionally, VSIs are adopted to indicate how close of the system to voltage collapse where the more value of the VSIs, the closer of the system to voltage collapse. Thus, the summation of the line VSIs for all transmission lines are aimed to be minimized in order to enhance the whole system voltage stability. The performance of considering the VSIs as the objective function is compared to that of the cost objective. Hence, four objective functions consisting of fuel cost function and the summation of three VSIs are selected as the objective function as follows:

\section{III.I.I Base case: generation cost function}

Generation cost is the base objective of power plants to be minimized. The function is formulated as shown below.

GenCost $=\sum_{i=1}^{N_{g e n}}\left(a_{i}+b_{i} P_{g i}+c_{i} P_{g i}^{2}\right)$ where $N_{\text {gen }}$ is the number of generators, $a_{i}, b_{i}, c_{i}$ are generation cost coefficients of the $i^{\text {th }}$ generator and $P_{g i}$ is the active power generation of the $i^{\text {th }}$ generator.

\section{III.I.II Summation Lmn of all lines}

The Lmn value of each transmission line can be computed by using (1), and the summation of Lmn values of all lines is focused as the objective function to be minimized to enhance the whole system stability. This Lmn objective is expressed as the provided equation.

$\mathrm{Lmn}_{\text {total }}=\sum_{i=1}^{N_{L}} \mathrm{Lmn}_{i}$

where $N_{L}$ is the number of transmission line and $\mathrm{Lmn}_{i}$ is the $\mathrm{Lmn}$ value at the $i^{\text {th }}$ bus.

\section{III.I.III Summation VCPI of all lines}

Similar to the summation of Lmn, the values of VCPI are evaluated for the entire lines in the system by (2). Thus, the voltage stability of the whole system can be enhanced by considering the summation of the VCPI as the objective function that can be presented below.

$\mathrm{VCPI}_{\text {total }}=\sum_{i=1}^{N_{L}} \mathrm{VCPI}_{i}$

where $\mathrm{VCPI}_{i}$ is the VCPI value at the $i^{\text {th }}$ bus.

\section{III.I.IV Summation FVSI of all lines}

To intensify the voltage stability of the whole system, the summation of FVSI of all transmission lines is imposed as the objective function to be minimized. The FVSI value can be found by (6). So, the objective function is presented as the equation presented below.

$\mathrm{FVSI}_{\text {total }}=\sum_{i=1}^{N_{L}} \mathrm{FVSI}_{i}$

where $\mathrm{FVSI}_{i}$ is the FVSI value at the $i^{\text {th }}$ bus.

\section{III.II Traditional constraints}

In power systems, the traditional constraints can be presented as follows [35]:

\section{III.II.I Equality constraints}

The equality constraints are the active and reactive power balance at each bus that are load flow equations expressed as shown below. 
International Journal of Engineering Research and Technology. ISSN 0974-3154, Volume 13, Number 7 (2020), pp. 1635-1648

(C) International Research Publication House. https://dx.doi.org/10.37624/IJERT/13.7.2020.1635-1648

$P_{g i}=P_{d i}+V_{i} \sum_{j=1}^{N_{b u s}} V_{j}\left(G_{i j} \cos \left(\theta_{i j}\right)+B_{i j} \sin \left(\theta_{i j}\right)\right)$

$Q_{g i}=Q_{d i}+V_{i} \sum_{j=1}^{N_{b u s}} V_{j}\left(G_{i j} \sin \left(\theta_{i j}\right)-B_{i j} \cos \left(\theta_{i j}\right)\right)$

where $i=1,2, \ldots, N_{b u s}, N_{b u s}$ is the number of buses, $P_{g i}$ and $Q_{g i}$ are the active and reactive power generations at the $i^{\text {th }}$ bus. $P_{d i}$ and $Q_{d i}$ are the active and reactive power demands at the $i^{\text {th }}$ bus, $V i$ and $V j$ are the voltage magnitudes at the $i^{\text {th }}$ and $j^{\text {th }}$ buses, $\theta_{i j}$ is the voltage phase angle difference between the $i^{\text {th }}$ and $j^{\text {th }}$ buses, and $G_{i j}$ and $B_{i j}$ are the transfer conductance and susceptance between the $i^{\text {th }}$ and $j^{\text {th }}$ buses.

\section{III.II.II Inequality constraints}

The state and control variables of the problem are limited by the inequality constraints to ensure system and assure device security. The inequality constraints are presented as given below.

$$
\begin{aligned}
& P_{g i \min } \leq P_{g i} \leq P_{g i \max } \quad i=1,2, \ldots, N_{g e n} \\
& Q_{g i \min } \leq Q_{g i} \leq Q_{g i \max } \quad i=1,2, \ldots, N_{g e n} \\
& \left|S_{l}\right| \leq S_{l \max } \\
& V_{i \min } \leq V_{i} \leq V_{i \max } \quad i=1,2, \ldots, N_{L} \\
& Q_{c i \min } \leq Q_{c i} \leq Q_{c i \max } \quad i=1,2, \ldots, N_{c a p} \\
& T_{i \min } \leq T_{i} \leq T_{i \max } \quad i=1,2, \ldots, N_{t r a n}
\end{aligned}
$$

where $P_{\text {gimax }}$ and $P_{\text {gimin }}$ are the maximum and minimum generator active powers at the $i^{\text {th }}$ bus, $Q_{\text {gimax }}$ and $Q_{\text {gimin }}$ are the maximum and minimum generator reactive powers at the $i$ th bus, $S_{l}$ and $S_{\text {lmax }}$ are the line flow power and its maximum value between buses $i$ and $j, V_{\text {imax }}$ and $V_{\text {imin }}$ are the maximum and minimum voltage magnitudes at the $i^{\text {th }}$ bus, $Q_{c}, Q_{\text {cimax }}$ and $Q_{\text {cimin }}$ are the shunt compensation capacitors its maximum and minimum values at the $i^{\text {th }}$ bus, $T_{i}, T_{\text {imax }}$ and $T_{\text {imin }}$ are the transformer tap ratios and its maximum and minimum values at the $i^{\text {th }}$ bus, $N_{c a p}$ is the number of compensation capacitors, and $N_{\text {tran }}$ is the number of transformer taps.

\section{III.III Voltage stability constraints}

From the inequality constraints, the voltage of each line is normally limited for the security reason. However, in the heavily loaded condition and line outage contingency, only the voltage limit constraint is inadequate to assure an acceptable voltage stability level. Thus, the voltage stability constraint is also defined as another inequality constraint, so that the maximum VSI value is restricted when a system is operating close to the limit and operators can move the system to a secure point. In this work, three VSIs are individually added to the inequality constraints as the presented equations.

$$
\begin{aligned}
& \mathrm{Lmn}_{\text {max }} \leq \mathrm{Lmn}_{\text {limit }} \\
& \mathrm{VCPI}_{\text {max }} \leq \mathrm{VCPI}_{\text {limit }}
\end{aligned}
$$

$\mathrm{FVSI}_{\max } \leq \mathrm{FVSI}_{\text {limit }}$

where $\mathrm{VSI}_{\text {limit }}\left(\mathrm{Lmn}_{\text {limit }}, \mathrm{VCPI}_{\text {limit }}\right.$ and $\left.\mathrm{FVSI}_{\text {limit }}\right)$ is a set threshold value of the VSI to guarantee a system stability level and VSI $\mathrm{Vax}_{\max }$ $\left(\mathrm{Lmn}_{\max }, \mathrm{VCPI}_{\max }\right.$ and $\left.\mathrm{FVSI}_{\max }\right)$ is a maximum value of the VSI which can be determined by the following equation.

$$
\mathrm{VSI}_{\text {max }}=\max \left(\mathrm{VSI}_{i}\right), i=1,2, \ldots, N_{L}
$$

\section{LINE VOLTAGE STABILITY INDICES BASED ON OPTIMAL POWER FLOW PROBLEM}

To enhance the system stability, VSIs are adopted as the added constraint and the objective functions. Then, the OPF problem is solved to find optimal solutions for each case. In this work, the OPF problems were solved by using SSA. The SSA and the application of incorporating VSIs to the OPF problems are explained as the following subsections:

\section{IV.I Salp Swarm Algorithm}

SSA is a recent metaheuristic optimization algorithm which is inspired by the swarming behavior of salps in the deep ocean [28]. Salps normally move as a swarm traditionally called salp chain for reaching better motion using fast coordinated moves and foraging [36].

The mathematic formulation of salp chains can be achieved by firstly separating salp population as a leader and followers. The leader is the first salp of the chain, and leading the chain is its role. The remaining salps of the chain are followers moving by following each other. The position of salps is imposed in the ndimensional search space where $n$ is the number of control variables of a design problem. Then, two-dimensional matrix $x$ is defined to keep the position. The position of the leader is mathematically formulated as the given equation.

$x_{j}^{1}=\left\{\begin{array}{lll}F_{j}+c_{1}\left(\left(u b_{j}-l b_{j}\right) c_{2}+l b_{j}\right) & \text { if } & c_{3} \geq 0 \\ F_{j}-c_{1}\left(\left(u b_{j}-l b_{j}\right) c_{2}+l b_{j}\right) & \text { if } & c_{3}<0\end{array}\right.$

where $x_{j}{ }^{1}$ is the leader position in the $j^{\text {th }}$ dimension, $F_{j}$ is the food source position which is the target of the swarm in the $j^{\text {th }}$ dimension, $u b_{j}$ is the upper bound of the $j^{\text {th }}$ dimension, $l b_{j}$ is the lower bound of the $j^{\text {th }}$ dimension, $c_{2}, c_{3}$ are the random numbers uniformly generated between 0 to 1 , and $c_{1}$ is an important coefficient, adopted to balance exploration and exploitation phases of the optimization process, of the leader and can be found as the provided equation.

$c_{1}=2 \exp \left\lfloor-\left(\frac{4 \text { Iter }}{\text { Iter }_{\text {max }}}\right)^{2}\right\rfloor$

where Iter is the current iteration and Iter $_{\max }$ is the number of maximum iteration. 
International Journal of Engineering Research and Technology. ISSN 0974-3154, Volume 13, Number 7 (2020), pp. 1635-1648

(C) International Research Publication House. https://dx.doi.org/10.37624/IJERT/13.7.2020.1635-1648

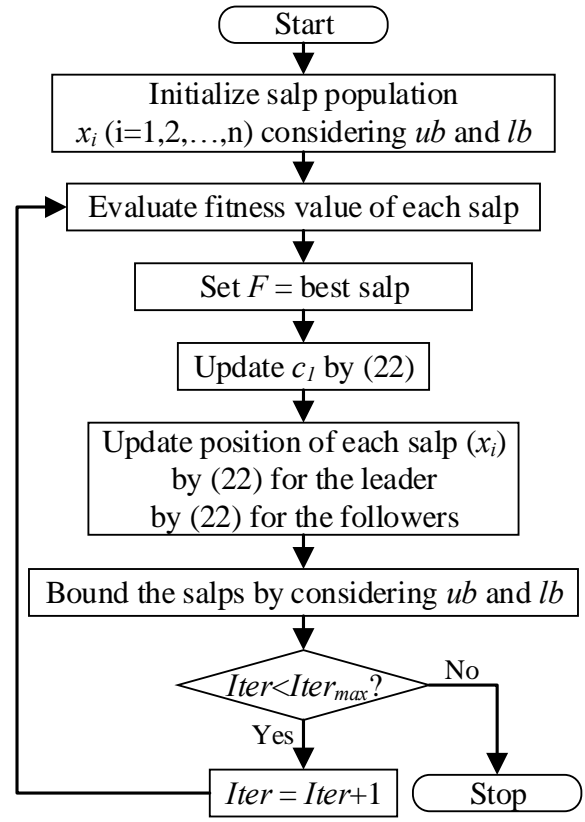

Fig. 2. Flowchart of SSA

The position of the followers is updated according to the Newton's law of motion which was proved in [28]. So, the following equation is computed to update the followers' position.

$$
x_{j}^{i}=\frac{1}{2}\left(x_{j}^{i}+x_{j}^{i-1}\right)
$$

where $i \geq 2$ indicating the followers and $x_{j}{ }^{\mathrm{i}}$ is the position of the $i^{\text {th }}$ follower in the $j^{\text {th }}$ dimension

The flowchart of the SSA is presented in Fig 2.

\section{IV.II Application of the approach}

The considered line VSIs are selected as part of the constraint and also objective function to prevent a system from voltage collapse and improve the system stability, respectively. Once the initialized search agents are generated, one option is chosen from minimizing cost, setting VSI as a constraint or imposing VSI as the objective function. When the first option (cost function) is adopted as the objective function, which is a base case for this study, cost function is calculated by (10) and the traditional constraints (Eqs. (14)-(21)) are applied. If the VSI is set as a constraint, which is the second option, cost function is computed as the objective function and the traditional constraints (Eqs. (14)-(21)) together with the VSI constraint (Eq. (22) for Lmn, Eq. (23) for VCPI or Eq. (24) for FVSI) are employed. If the last option is selected, VSI is assigned as the objective function. Then, the summation of VSI is evaluated by (11) for Lmn, (12) for VCPI or (13) for FVSI, and the traditional constraints (Eqs. (14)-(21)) are used. After choosing one option and evaluating the equations as mentioned before, the OPF problem is solved by SSA. This process is operated until

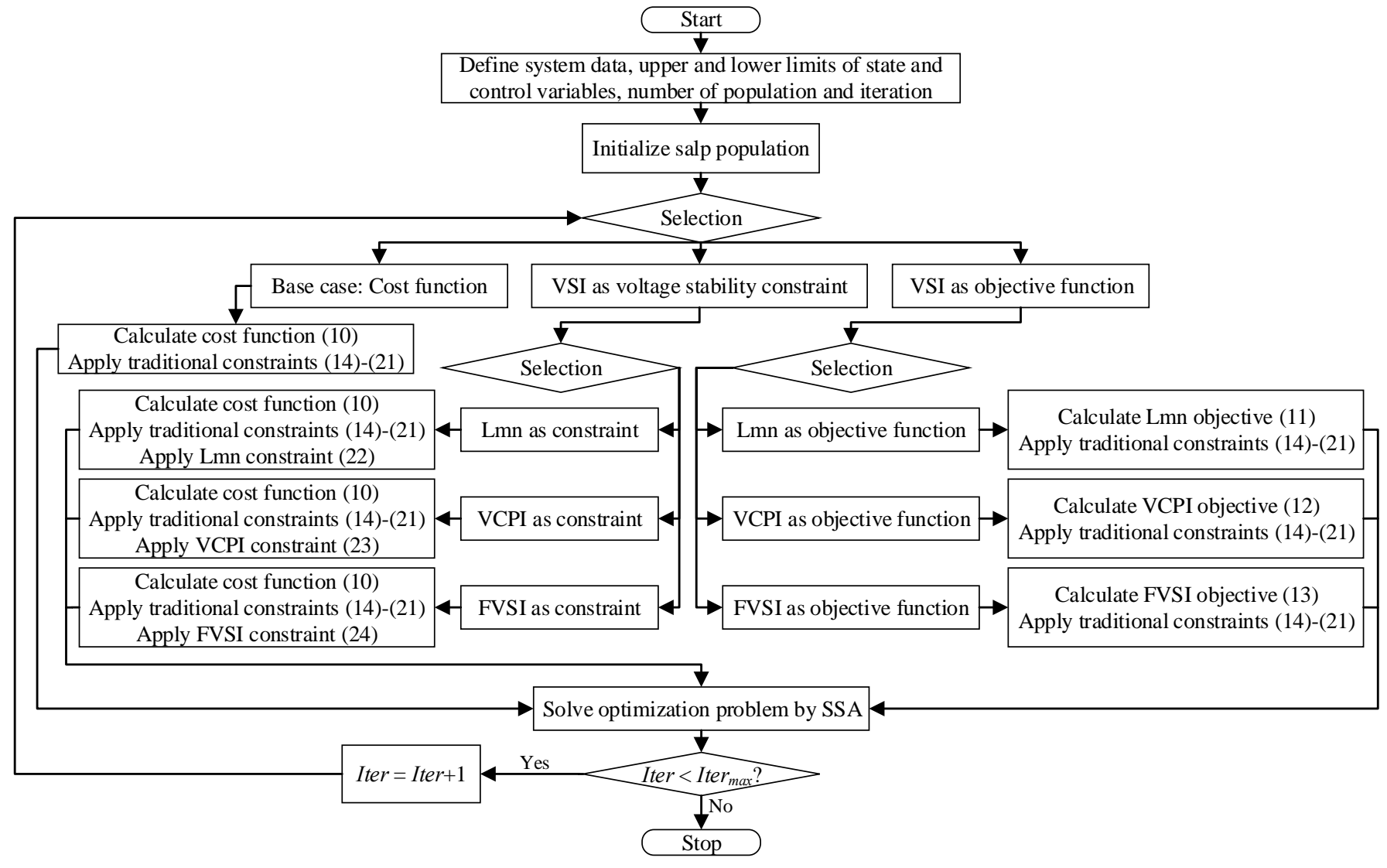

Fig. 3. Flowchart of the VSI approach 
International Journal of Engineering Research and Technology. ISSN 0974-3154, Volume 13, Number 7 (2020), pp. 1635-1648

(C) International Research Publication House. https://dx.doi.org/10.37624/IJERT/13.7.2020.1635-1648

Table 1. Simulation results in heavily loaded situation for IEEE 30-bus system

\begin{tabular}{|l|c|c|c|c|c|c|c|}
\hline & \multicolumn{7}{|c|}{ Objective functions } \\
\cline { 2 - 8 } & Cost & Lmncon & VCPIcon & FVSIcon & Lmnobj & VCPIobj & FVSIobj \\
\hline Cost $(\$ / \mathrm{h})$ & 1268.5738 & 1269.1244 & 1272.6130 & 1269.0111 & 1296.5159 & 1302.4506 & 1294.4194 \\
\hline Qgen (MVAR) & 178.4510 & 164.4603 & 155.6071 & 171.0796 & 139.2880 & 137.6900 & 140.3301 \\
\hline Loss (MW) & 14.8128 & 14.5126 & 14.5112 & 14.5560 & 13.7381 & 13.7333 & 13.8010 \\
\hline Lindex & 0.1938 & 0.1913 & 0.1919 & 0.1914 & 0.1860 & 0.1824 & 0.1847 \\
\hline
\end{tabular}

reaching the stop criteria. The application of the approach is depicted in Fig 3.

\section{SIMULATION RESULTS}

The comparative study of the static line VSIs was investigated on the IEEE 30-bus and 118-bus systems. For each test system, each case study was operated for 30 independent runs. The IEEE 30-bus system consists of 6 generators, 4 transformers and 41 transmission lines. The detailed data can be found in [37]. The other test system which is the IEEE 118-bus system comprises of 54 generators, 9 transformers, and 186 transmission lines. The system data can be obtained from [38].

The VSIs are individually incorporated to the OPF problem in two approaches including considering it as added constraint and as the objective function to be minimized. These two approaches aim to reduce active power loss of a system and also enhance the voltage stability by minimizing line loss and maximizing system loadability. The maximum loadability of each case is evaluated by generating PV curves using continuation power flow (CPF) [39]. To further verify the ability of the line VSIs to enhance the system voltage stability, the values of the considered VSIs can be evaluated. However, the values of each VSI (Lmn, VCPI and FVSI) cannot be compared to each other since they all have the difference formulations. So, to compare the system voltage stability improvement, L-index is calculated as an independent indicator. L-index is a bus VSI which has been widely and popularly used to indicate the critical bus of the system leading to the voltage collapse [9]. The L-index value is between 0 (no-load condition) and 1 (voltage collapse), so the less L-index value, the more efficiency of the VSIs to improve system voltage stability. To investigate the performance of using VSIs as the added constraint or the objective function, the simulation is assessed in two situations comprising of heavy load and line outage contingency for each test system. The simulation results for each case are presented as follows:

\section{V.I Heavily loaded situation for the IEEE 30-bus system}

In the heavily loaded situation, the real and reactive powers of each bus of the IEEE 30-bus system are raised to 1.4 times base load. Each VSI is imposed as part of the constraint to restrict the VSI value within its limit and move the system far from the voltage collapse when the system are heavily loaded. In this situation, the summation of each VSI is employed as the objective function to be minimized to enhance the overall system stability. The simulation results of these two approaches are presented in Table 1, and the comparison can be investigated from Fig 4.

\section{V.I.I VSIs as part of the constraint}

In this part, the studied VSIs consisting of Lmn, VCPI and FVSI were individually added as part of the constraint and the results are compared to the base case. The simulation results displayed in Table 1 and Fig 4 show that adding VCPI as part of the constraint could mostly reduce reactive power generation by $12.80 \%$ following by Lmn and FVSI which are $7.84 \%$ and $4.13 \%$ decrease, respectively. Similarly, the most and second most percentage reductions of loss were also obtained by adding VCPI (2.04\% reduction) and Lmn (2.03\% reduction) as part of the constraint, respectively, and FVSI could decrease the loss by $1.73 \%$ reduction. By considering L-index value to analyze the system voltage stability enhancement in this situation, when combining Lmn, FVSI and VCPI as part of the constraint, Lindex values were minimized with a percentage of $1.29 \%$, $1.25 \%$ and $0.96 \%$, respectively. However, the generation costs were slightly increased by $0.32 \%$ for VCPI, $0.04 \%$ for $\mathrm{Lmn}$ and

\section{Cost $\square$ Lmncon $\square$ VCPIcon $\square$ FVSIcon $\square$ Lmnobj $\square$ VCPIobj $\square$ FVSIobj}
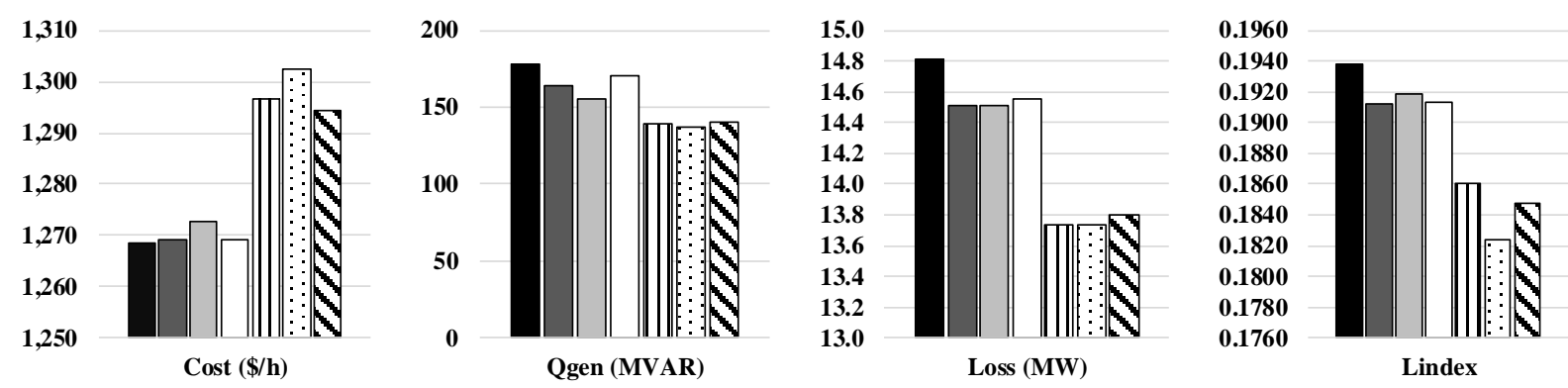

Fig 4 Comparison of the simulation results in heavily loaded situation for IEEE-30 bus system 
Table 2 Maximum loadability results in heavily loaded situation for IEEE 30-bus system

\begin{tabular}{|c|c|}
\hline Objective functions & Maximum loadability (MW) \\
\hline Cost & 875.4400 \\
\hline Lmncon & 914.5108 \\
\hline VCPIcon & 914.5722 \\
\hline FVSIcon & 913.9332 \\
\hline Lmnobj & 915.0086 \\
\hline VCPIobj & 925.5991 \\
\hline FVSIobj & 915.6408 \\
\hline
\end{tabular}

$0.03 \%$ for FVSI. Note that the cost is less grown up when the load is less increased from the base load.

To analyze the maximum loadability of a system when merging each VSI as part of the constraint, a continuation power flow $(\mathrm{CPF})$ is employed to generate a PV curve where the active load power can be traced until reaching the voltage collapse (critical point). So, the maximum loadability of each case is shown in Table 2 and Fig 5. Comparing to the base case, the maximum loadability was improved about $4.47 \%$ when using VCPI as part of the constraint following by Lmn (4.46\%) and FVSI (4.40\%), respectively.

\section{V.I.II VSIs as the objective function}

The simulation results of selecting each VSI comprising of Lmn, VCPI and FVSI as the objective function are shown in Table 1 and Fig 4 and compared to the base case. It is observed that the reactive power generations were reduced around $22.84 \%$, $21.95 \%$ and $21.36 \%$ when choosing VCPI, Lmn and FVSI as the objective function, respectively. The transmission line loss values when considering VCPI, Lmn and FVSI as the objective function were decreased by $7.29 \%, 7.25 \%$ and $6.83 \%$. When noticing the L-index values, they were moved down approximately $5.86 \%$ for VCPI, $4.67 \%$ for FVSI and $4.00 \%$ for $\mathrm{Lmn}$. On the other hand, the generation costs of VCPI, Lmn and FVSI met the percentage increase by $2.67 \%, 2.20 \%$ and $2.04 \%$, respectively.

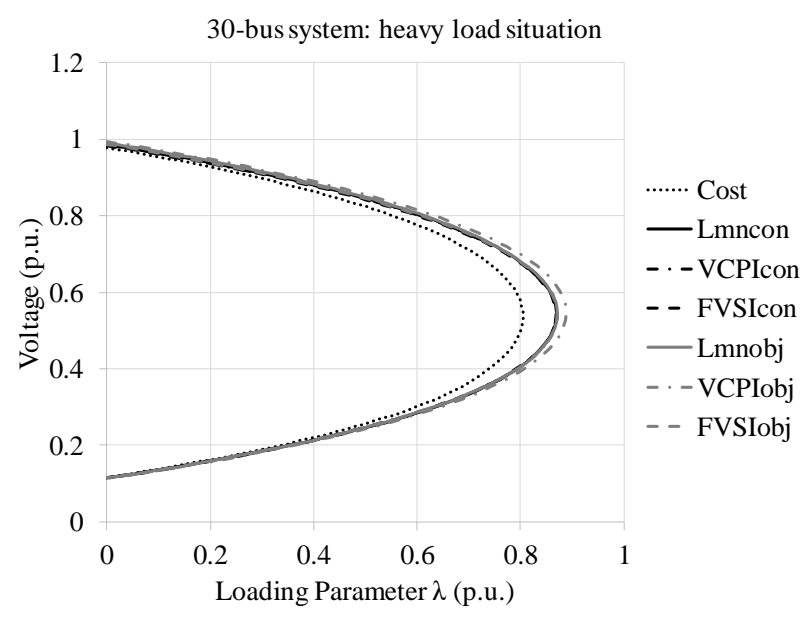

Fig 5 Comparison of the PV curves of all cases in heavily loaded situation for IEEE 30-bus system
The PV curves representing maximum loadability and the values when defining VSIs as the objective function are demonstrated in Table 2 and Fig 5. The maximum loadability values were enhanced $5.73 \%, 4.59 \%$ and $4.52 \%$ for VCPI, FVSI and Lmn, respectively, compared to the base case.

In the heavily loaded situation, it is found that using VSIs as the objective function could better reduce transmission line loss and enhance voltage stability (less L-index value) than those of the adding VSIs as part of the constraint and base case. Moreover, combining VSIs as part of the constraint also provides less transmission loss and further improves voltage stability improvement than those of the base case even though they are worse than the VSI objectives. The maximum loadability values of all VSI objective functions, especially VCPI objective, are

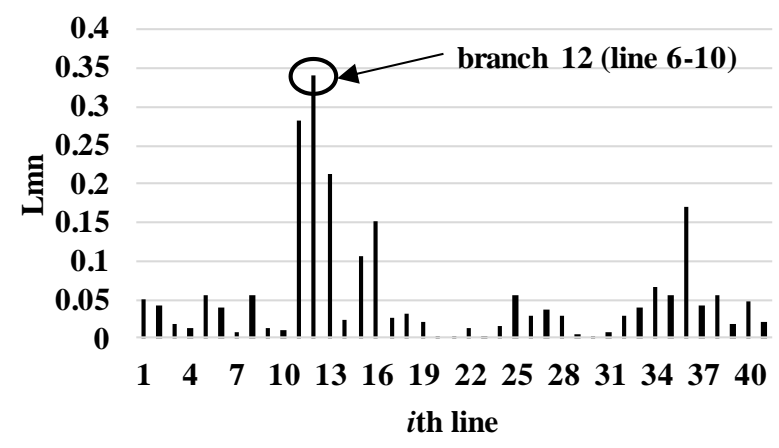

(a) Lmn

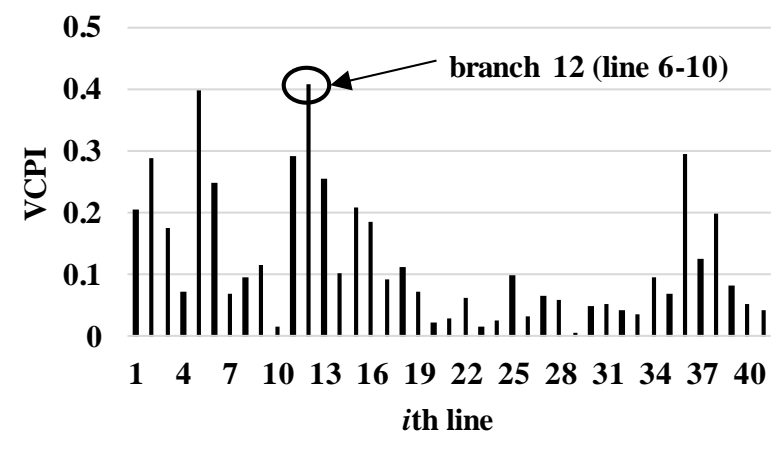

(b) VCPI

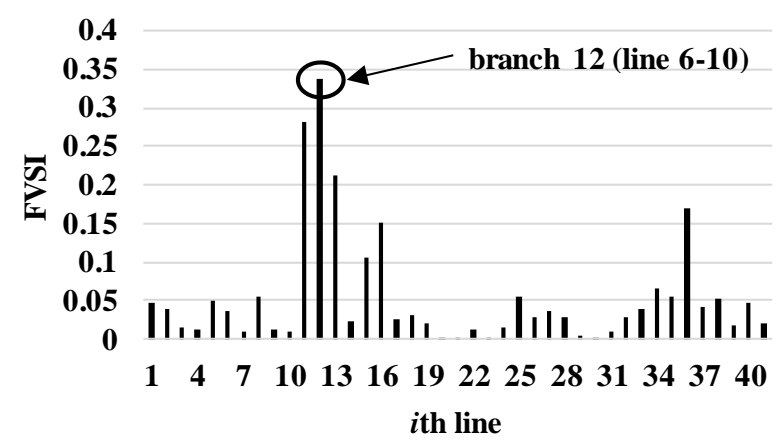

(c) FVSI

Fig 6 VSI values of each line in heavily loaded situation for IEEE 30-bus system 
International Journal of Engineering Research and Technology. ISSN 0974-3154, Volume 13, Number 7 (2020), pp. 1635-1648

(C) International Research Publication House. https://dx.doi.org/10.37624/IJERT/13.7.2020.1635-1648

Table 3 Simulation results in line outage contingency situation for IEEE 30-bus system

\begin{tabular}{|l|c|c|c|c|c|c|c|}
\hline & \multicolumn{7}{|c|}{ Objective functions } \\
\cline { 2 - 9 } & Cost & Lmncon & VCPIcon & FVSIcon & Lmnobj & VCPIobj & FVSIobj \\
\hline Cost $(\$ / \mathrm{h})$ & 802.2692 & 802.3133 & 802.3243 & 802.3735 & 841.1208 & 857.9211 & 821.7957 \\
\hline Qgen (MVAR) & 98.1348 & 98.3901 & 96.6662 & 94.2673 & 77.4516 & 80.3174 & 86.7355 \\
\hline Loss (MW) & 9.4600 & 9.3666 & 9.4479 & 9.4240 & 6.5101 & 5.6568 & 7.7174 \\
\hline Lindex & 0.13537 & 0.13535 & 0.1348 & 0.1347 & 0.1329 & 0.1346 & 0.1322 \\
\hline
\end{tabular}

more than those of the VSI constraints and the base case when the base case could obtain the minimum loadability. However, the generation cost of the VSI objectives are more than those of the VSI constraints and the base case that is acceptable when comparing to the decrease of transmission line loss and also the voltage stability enhancement for the heavily loaded situation.

\section{V.II Line outage contingency situation for the IEEE 30-bus system}

Normally, line outage contingency changes the system to undesirable operating conditions which could finally lead the system to the voltage collapse. Hence, the line outage contingency situation should be investigated to estimate how the voltage stability can be improved against the voltage collapse.

In this work, the line which has the highest value of VSIs is indicated as the weakest line and chosen as the line outage. From Fig 6, all considered VSIs including Lmn, VCPI and FVSI indicate that branch 12 connecting between buses 6 and 10 has the highest VSI values which is the weakest line for the IEEE 30-bus system where Figs 6(a), 6(b) and 6(c) represent Lmn, VCPI and FVSI values, respectively. So, branch 12 is chosen to evaluate the effect of the contingency condition.

In this situation, the focused VSIs were selected as part of the constraint and the objective function to improve the system voltage stability and prevent the voltage collapse from the undesirable conditions. Table 3 presents the simulation results of each case study, and the comparison results can be observed in Fig 7.

\section{V.II.I VSIs as part of the constraint}

From Table 3 and Fig 7, it is noticeable that considering VSIs as part of the constraint was able to slightly improve the transmission loss and voltage stability compared to the base case in the line outage contingency situation. The reactive power generations of adding FVSI, VCPI and Lmn as part of the constraint were minimized by $4.19 \%, 1.75 \%$ and $0.26 \%$, respectively. Furthermore, incorporating Lmn, FVSI and VCPI as part of the constraint resulted in a reduction of $0.99 \%, 0.38 \%$ and $0.13 \%$ of transmission line loss, respectively. By investigating the L-index values, it was decreased representing an enhancement of the voltage stability of $0.52 \%$ (FVSI), $0.44 \%$ (VCPI) and $0.01 \%(\mathrm{Lmn})$. Conversely, the generation costs were grown up by roughly $0.01 \%$ for all VSIs.

By evaluating the maximum loadability of integrating VSIs as part of the constraint in the line outage contingency situation, the PV curves were generated as displayed in Fig 8. The maximum loadability values of each VSI constraint were marginally improved from the base case of around $0.13 \%$, $0.05 \%$ and $0.04 \%$ for Lmn, FVSI and VCPI, respectively, indicating an improvement of the maximum loadability.

\section{V.II.II VSIs as the objective function}

To further enhance the system stability, the VSIs were considered as the objective function, and the simulation results can be found in Table 3 and Fig 7. When comparing to the base case, both reactive power generations and transmission losses of the VSI objectives are significantly reduced. The reactive power generations of Lmn, VCPI and FVSI were moved down with a percentage reduction of $21.28 \%$ (Lmn), $18.37 \%$ (VCPI) and $11.85 \%$ (FVSI). In addition, the transmission line loss of VCPI, Lmn and FVSI were dropped by $40.20 \%, 31.18 \%$ and $18.42 \%$, respectively. The L-index values, which represent the voltage stability improvement, of FVSI, Lmn and VCPI were improved

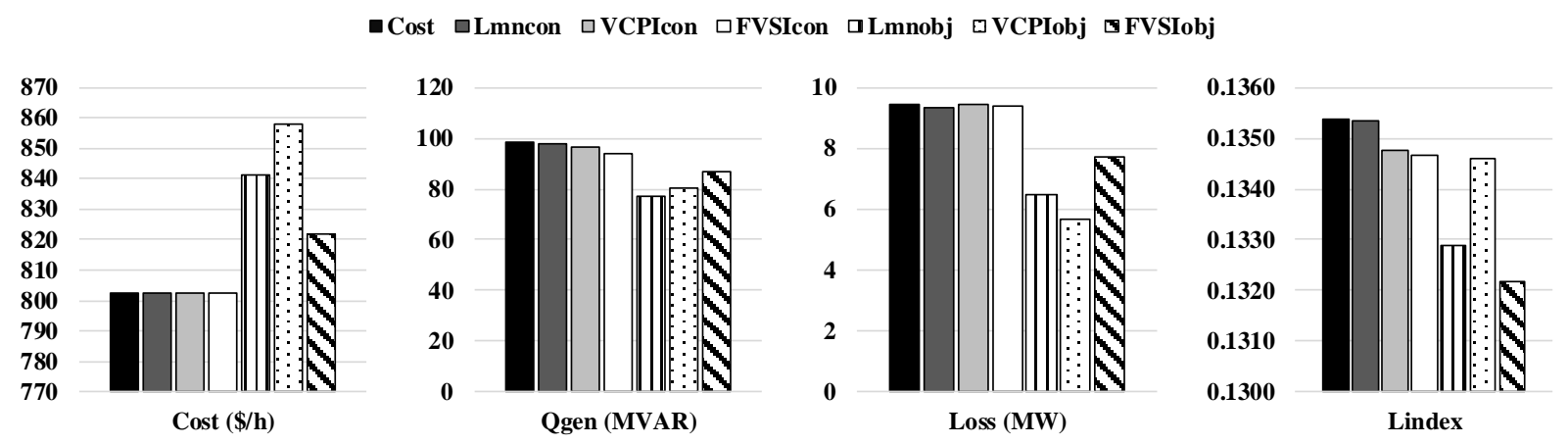

Fig 7 Comparison of the simulation results in line outage contingency situation for IEEE-30 bus system 
Table 4 Maximum loadability results in line outage contingency situation for IEEE 30-bus system

\begin{tabular}{|c|c|}
\hline Objective functions & Maximum loadability (MW) \\
\hline Cost & 869.1577 \\
\hline Lmncon & 870.3054 \\
\hline VCPIcon & 869.5099 \\
\hline FVSIcon & 869.5986 \\
\hline Lmnobj & 874.6653 \\
\hline VCPIobj & 885.7792 \\
\hline FVSIobj & 876.5479 \\
\hline
\end{tabular}

by $2.35 \%, 1.85 \%$ and $0.56 \%$, respectively. In contrast, the VSI objective functions resulted $6.94 \%, 4.84 \%$ and $2.43 \%$ increase in generation cost for VCPI, Lmn and FVSI, respectively.

When the load demand is gradually raised until the critical point, the maximum loadability cuvres of cooperating VSIs as the objective function are presented in Fig 8. The maximum loadability values of all VSIs were enhanced from the base case with a precentage minimization of $1.91 \%$ (VCPI), $0.85 \%$ (FVSI) and $0.63 \%$ (Lmn) as shown in Table 4.

By considering the line outage contingency situation, selecting VSIs as the objective function provides considerably better loss minimization and votlage stability improvement than those of the base case and the merging VSIs as part of the constraint. VCPI objective could decrease loss up to $40.20 \%$ followed by about $31 \%$ for Lmn and $18 \%$ for FVSI compared to the base case although all considered VSI constraints could only obtain less $1 \%$ of loss reduction than the base case. Moreoever, the percentage minimization of L-index, representing the voltage stability improvement, and the maximum loadability are enhanced by employing VSI constraints and particularly by adopting VSI objectives. Finally, the generation costs of VSI constraints and objectives are slightly grown up, and it is worth to increase the generation cost for the voltage stability enhancement and loss reduction in the line outage contingency situation.

\section{V.III Heavily loaded situation for the IEEE 118-bus system}

To verify the performance of the VSI constraint and VSI objective to enhance the system stability in the heavily loaded situation, a larger system which is the IEEE 118-bus system was tested. Similar to the IEEE 30-bus system, the real power and

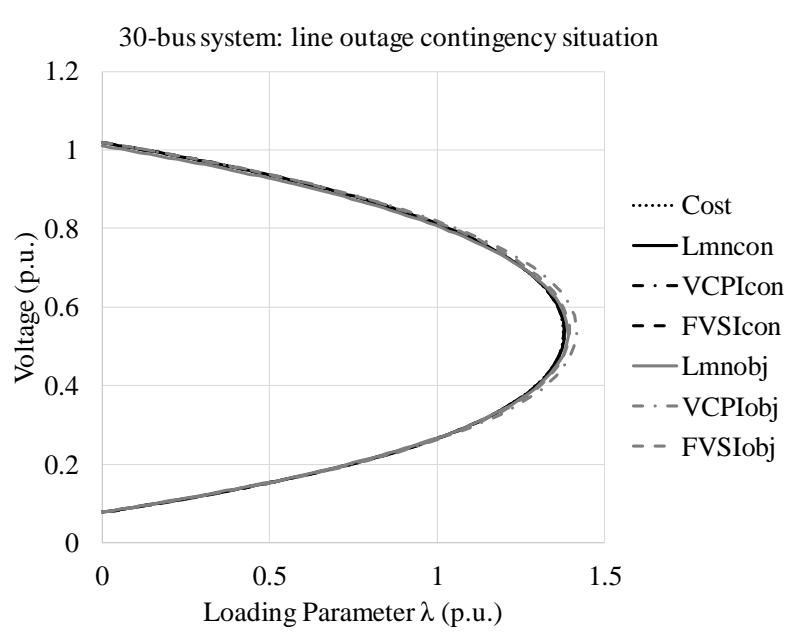

Fig 8 Comparison of the PV curves of all cases in line outage contingency situation for IEEE 30-bus system

reactive power demands of each bus are moved up to 1.4 times base load. The simulation results for this IEEE 118-bus system are presented in Table 5, compared as in Fig 9 and explained as the following subsections.

\section{V.III.I VSIs as part of the constraint}

From the results in Table 5 and Fig 9 compared to the base case, when VSIs are combined as part of the constraint in the heavily loaded situation, the line loss is obviously reduced and the voltage stability is significantly improved by considering Lindex value. FVSI, VCPI and Lmn constraints could obtain the minimization of reactive power generations corresponding to the $3.92 \%, 2.72 \%$ and $2.45 \%$ minimization, respectively. The transmission line loss were also decreased by $7.71 \%$ (FVSI), $5.84 \%$ (Lmn) and $5.73 \%$ (VCPI). The voltage stability was enhanced by the reduction of L-index values with a percentage reduction of $8.08 \%$ for VCPI, $6.13 \%$ for FVSI and $3.51 \%$ for Lmn. On the contrary, the VSI constraints caused the light increase of the generation cost by $4.93 \%, 3.61 \%$ and $1.43 \%$ provided by Lmn, FVSI and VCPI constraints, respectively.

The PV curves representing the maximum loadability before the voltage collapse of each VSI constraint are plotted as in Fig 10 and the values are given in Table 6 . VCPI constraint provided the most maximum loadability by $3.27 \%$ more than the base

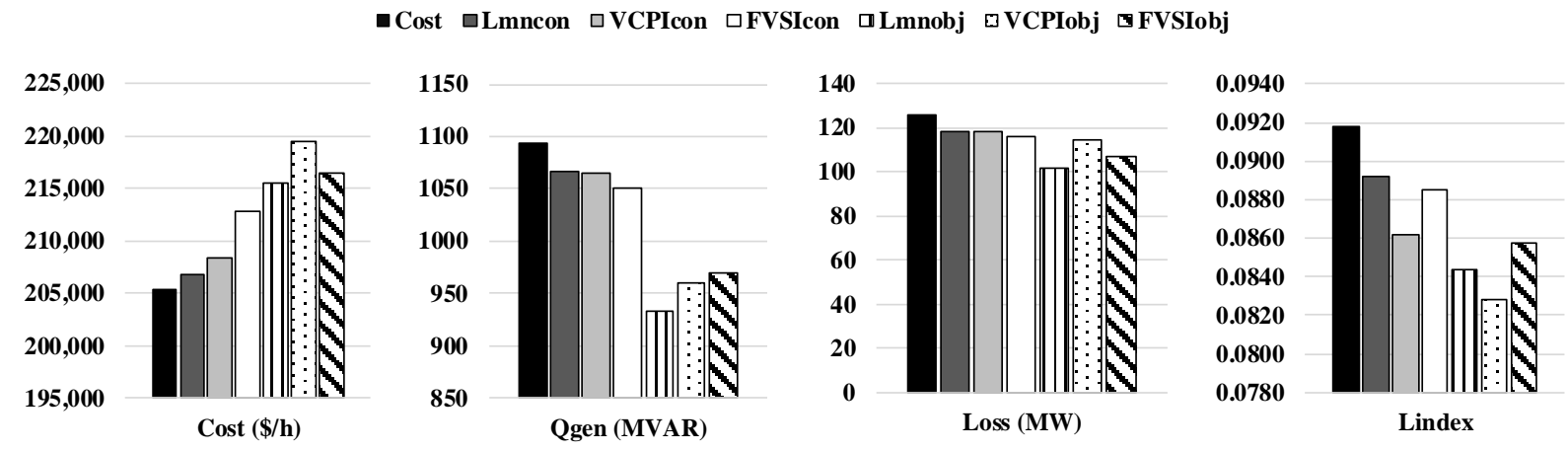

Fig 9 Comparison of the simulation results in heavily loaded situation for IEEE-118 bus system 
International Journal of Engineering Research and Technology. ISSN 0974-3154, Volume 13, Number 7 (2020), pp. 1635-1648

(C) International Research Publication House. https://dx.doi.org/10.37624/IJERT/13.7.2020.1635-1648

Table 5 Simulation results in heavily loaded situation for IEEE 118-bus system

\begin{tabular}{|l|c|c|c|c|c|c|c|}
\hline & \multicolumn{7}{|c|}{ Objective functions } \\
\cline { 2 - 8 } & Cost & Lmncon & VCPIcon & FVSIcon & Lmnobj & VCPIobj & FVSIobj \\
\hline Cost $(\$ / \mathrm{h})$ & 205411.71 & 206725.18 & 208358.68 & 212826.38 & 215548.41 & 219496.32 & 216512.50 \\
\hline Qgen (MVAR) & 1094.1443 & 1067.3302 & 1064.4350 & 1051.2734 & 933.2620 & 960.2508 & 970.2839 \\
\hline Loss (MW) & 125.9095 & 118.5616 & 118.6923 & 116.2072 & 101.8913 & 114.9651 & 106.8984 \\
\hline Lindex & 0.0918 & 0.0892 & 0.0861 & 0.0886 & 0.0844 & 0.0828 & 0.0858 \\
\hline
\end{tabular}

case followed by those of the FVSI and Lmn constraint which were $2.96 \%$ and $1.94 \%$ more than the base case, respectively, as shown in Table 6.

\section{V.III.II VSIs as the objective function}

In the heavily loaded situation, VSIs were adopted as the objective function, and the simulation results is shown as in Table 5 and Fig 9 compared to the base case. When Lmn was imposed as the objective function, the reactive power generation was reduced by $14.70 \%$ followed by VCPI and FVSI with the percentage reduction of $12.24 \%$ and $11.32 \%$, respectively. The transmission losses were decreased by $19.08 \%, 15.10 \%$ and $8.69 \%$ by imposing Lmn, FVSI and VCPI as the objective function, respectively. L-index values were minimized indicating an enhancement of the voltage stability of $9.74 \%$, $8.08 \%$ and $6.52 \%$, respectively, for VCPI, Lmn and FVSI. Nevertheless, employing VCPI, FVSI and Lmn as the objective function resulted $6.86 \%, 5.40 \%$ and $4.93 \%$ increases, respectively, in the generation costs.

To investigate the maximum loadability of using VSIs as the objective function, PV curves were generated as presented in Fig 10 where the maximum loadability values are provided in Table 6. It can be seen that the maximum loadability values generated by considering FVSI, VCPI and Lmn as the objective function were improved from the base case by $4.65 \%, 4.21 \%$ and $3.64 \%$, respectively.

For the heavily loaded situation in the IEEE 118-bus system, when compared to the base case, it can be noticed that transmission loss values are obviously decreased by using VSIs as part of the constraint and dramatically decreased by using VSIs as the objective function. Similarly, VSI constraints could significantly reduce the L-index values from the base case, and VSI objectives provide less L-index values, representing better voltage stability, than those of the VSI constraints and the base case, specifically VCPI and Lmn objectives reaching almost

Table 6 Maximum loadability results in heavily loaded situation for IEEE 118-bus system

\begin{tabular}{|c|c|}
\hline Objective functions & Maximum loadability (MW) \\
\hline Cost & 20905.3108 \\
\hline Lmncon & 21311.4982 \\
\hline VCPIcon & 21588.5341 \\
\hline FVSIcon & 21524.5194 \\
\hline Lmnobj & 21665.6333 \\
\hline VCPIobj & 21785.5410 \\
\hline FVSIobj & 21878.1974 \\
\hline
\end{tabular}

$10 \%$ L-index reduction in the IEEE 118-bus system. The PV curves generated by VSI objectives gave the higher maximum loadability values than those of the VSI constraints and the base case. In addition, the generation costs were reasonably raised in exchange to the voltage stability enhancement and loss reduction in the heavily loaded of this large system.

\section{V.IV Line outage contingency situation for the IEEE 118- bus system}

The IEEE 118-bus system was also employed to guarantee the performance of incorporating VSIs as the added constraint and the objective function in the line outage contingency situation. To identify the most critical line in this system, values of the Lmn, VCPI and FVSI were calculated for all lines. It can be seen from Fig 11 that branch 106 (connecting between buses 46 and 49 ) is the weakest line indicated by all of the considered VSIs which is apparent in Figs 11(a), 11(b) and 11(c). Therefore, branch 106 is chosen as the candidate line to investigate the effect of the line outage contingency.

The simulation results of adding VSIs as part of the constraint and considering VSIs as the objective function are demonstrated and compared with the generation cost objective (base case) as depicted in Table 7 and Fig 12, and the explanation and discussion are given as follows:

\section{V.IV.I VSIs as part of the constraint}

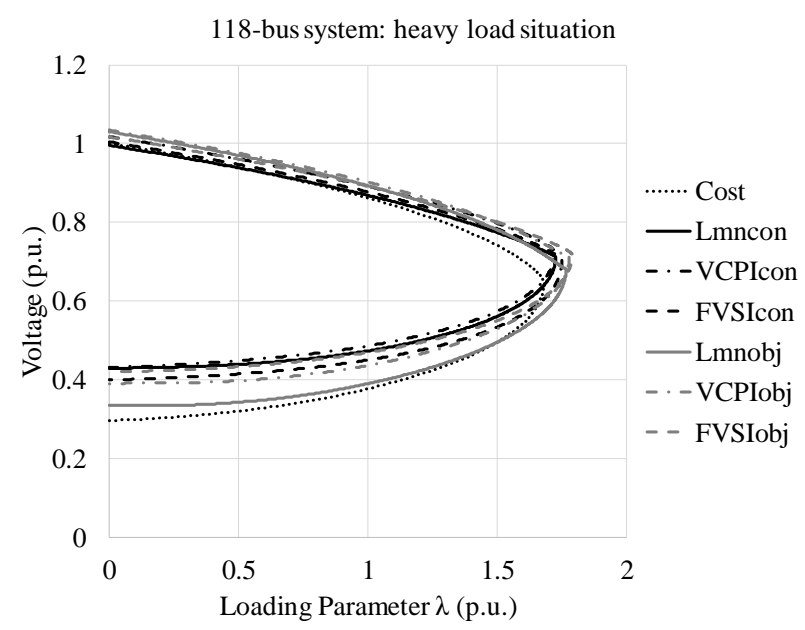

Fig 10 Comparison of the PV curves of all cases in heavily loaded situation for IEEE 118-bus system 


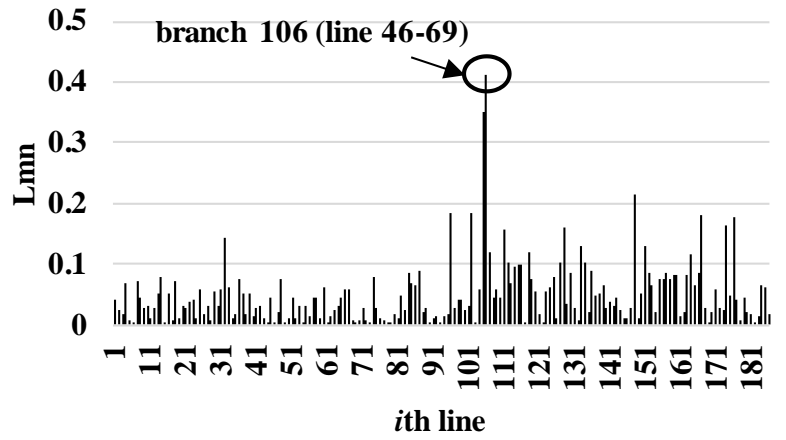

(a) Lmn

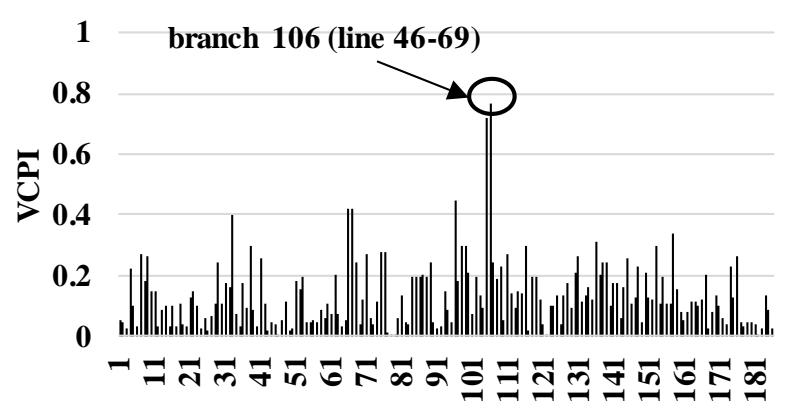

ith line

(b) VCPI

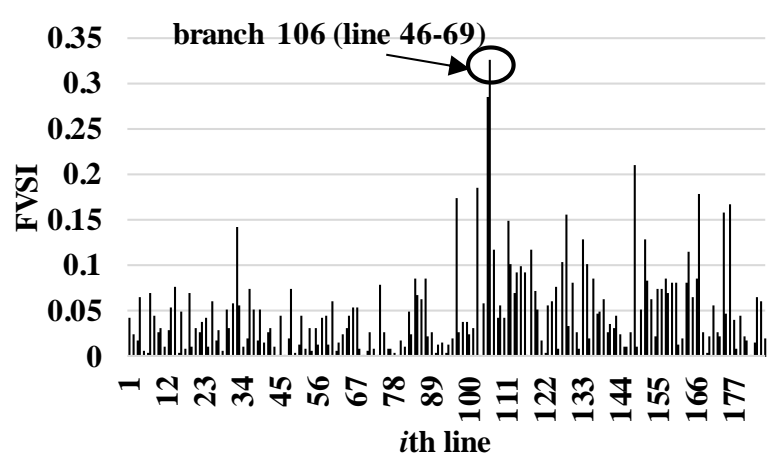

(c) FVSI

Fig 11 VSI values of each line in heavily loaded situation for IEEE 118-bus system

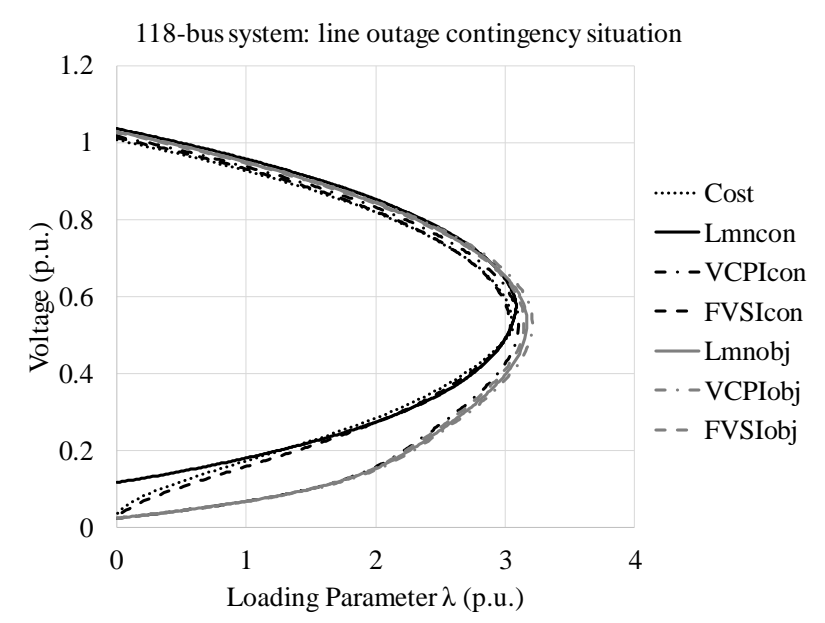

Fig 13 Comparison of the PV curves of all cases in line outage contingency situation for IEEE 30-bus system

By referring to Table 7 and Fig 12 compared to the base case, for the line outage contingency situation of the IEEE 118-bus system, adding VSIs as part of the constraint gave the reduction of reactive power generation corresponding to the $1.24 \%$ (FVSI), $0.92 \%$ (Lmn) and $0.73 \%$ (VCPI) reduction. The VSI constraints also resulted in a reduction of $18.06 \%$ for VCPI, $17.36 \%$ for FVSI and $14.54 \%$ for Lmn in transmission line losses. The system voltage stability was improved by $1.24 \%$, $0.92 \%$ and $0.73 \%$ for FVSI, VCPI and Lmn constraints, respectively, by noticing $\mathrm{L}$-index values. Conversely, the generation costs were moved up around $1.90 \%, 0.72 \%$ and $0.38 \%$ for VCPI, Lmn and FVSI constraints, respectively.

The PV curves of the VSI constraint were produced and plotted as in Fig 13, the maximum loadability values can be obtained from Table 8 . It is shown that the maximum loadability values were enhanced from the base case by $1.24 \%, 0.92 \%$ and $0.73 \%$ for VCPI, FVSI and Lmn, respectively.

\section{V.IV.II VSIs as the objective function}

When VSIs were considered as the objective function in the line outage contingency situation, the line loss and the system voltage stability are further improved compared to the base case as appeared in Table 7 and Fig 12. It is seen that Lmn, VCPI and

$\square$ Cost $\square$ Lmncon $\square$ VCPIcon $\square$ FVSIcon $\square$ Lmnobj $\square$ VCPIobj $\triangle$ FVSIobj
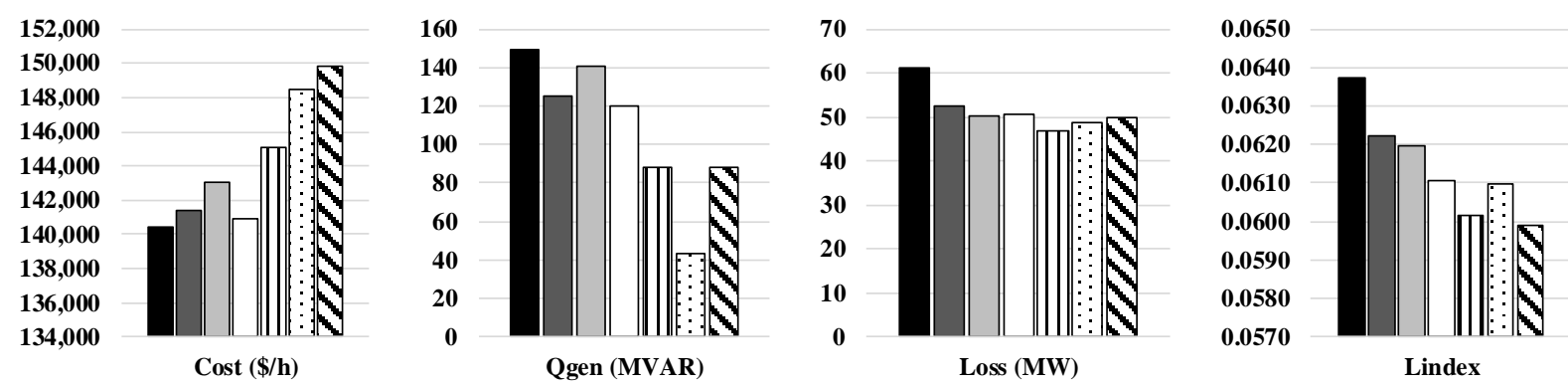

Fig 12 Comparison of the simulation results in line outage contingency situation for IEEE-118 bus system 
International Journal of Engineering Research and Technology. ISSN 0974-3154, Volume 13, Number 7 (2020), pp. 1635-1648

(C) International Research Publication House. https://dx.doi.org/10.37624/IJERT/13.7.2020.1635-1648

Table 7 Simulation results in line outage contingency situation for IEEE 118-bus system

\begin{tabular}{|l|c|c|c|c|c|c|c|}
\hline & \multicolumn{7}{|c|}{ Objective functions } \\
\cline { 2 - 8 } & Cost & Lmncon & VCPIcon & FVSIcon & Lmnobj & VCPIobj & FVSIobj \\
\hline Cost $(\$ / \mathrm{h})$ & 140406.20 & 141421.39 & 143073.00 & 140937.45 & 145070.10 & 148509.70 & 149894.87 \\
\hline Qgen (MVAR) & 149.5770 & 125.0365 & 140.6220 & 120.1051 & 87.8005 & 43.1510 & 88.3962 \\
\hline Loss (MW) & 61.4362 & 52.5016 & 50.3394 & 50.7717 & 46.7367 & 48.7920 & 49.7435 \\
\hline Lindex & 0.06375 & 0.06223 & 0.06199 & 0.06109 & 0.06015 & 0.06097 & 0.05988 \\
\hline
\end{tabular}

FVSI objectives resulted in a huge reduction of $71.15 \%, 41.30 \%$ and $40.90 \%$, respectively, in reactive power generation. The transmission loss values obtained by Lmn, VCPI and FVSI objectives were decreased by $23.93 \%, 20.58 \%$ and $19.03 \%$, respectively. FVSI, Lmn and VCPI objectives also gained a minimization of L-index values representing an improvement of the voltage stability of $6.07 \%, 5.64 \%$ and $4.36 \%$, respectively. However, the VSI objectives met the increase of generation costs by $6.76 \%$ (FVSI), $5.77 \%$ (VCPI) and $3.32 \%$ (Lmn). The increase of the generation costs represents the additional cost to enhance the system voltage stability and reduce the line loss.

To evaluate the maximum loadability of the system when VSIs were chosen as the objective function, the PV curves were produced and are shown in Fig 13, and the maximum loadability values can be obtained from Table 8 . It is observed that the maximum loadability of VCPI, Lmn and FVSI objectives were enhanced by $4.11 \%, 3.01 \%$ and $2.37 \%$, respectively, compared to the base case.

In the line outage contingency situation of the larger system which is the IEEE 118-bus system, choosing VSIs as part of the constraints and objectives functions could considerably minimize transmission line loss from the base case where VSI objective functions provide more percentage of minimization than those of the VSI constraints. The L-index values were also significantly decreased around $4.4-6.1 \%$ by using VSI objective and $2.4-4.2 \%$ by using VSI constraints from the base case that demonstrates the system voltage stability enhancement. By observing the critical point of the system, the maximum loadability values were improved about $3-4.1 \%$ for VSI objectives and $0.7-1.2 \%$ for VSI constraints compared to the base case. On the other hand, VSI objectives require more generation costs than those of the VSI constraints to more reduce transmission loss and improve system voltage stability.

In conclusion, different VSIs provided the best values in different terms, situations and systems. Moreover, by noticing

Table 8 Maximum loadability results in line outage contingency situation for IEEE 118-bus system

\begin{tabular}{|c|c|}
\hline Objective functions & Maximum loadability (MW) \\
\hline Cost & 23696.9841 \\
\hline Lmncon & 23868.9092 \\
\hline VCPIcon & 23991.0698 \\
\hline FVSIcon & 23914.1162 \\
\hline Lmnobj & 24409.5287 \\
\hline VCPIobj & 24670.5145 \\
\hline FVSIobj & 24259.1515 \\
\hline
\end{tabular}

the percentage improvement of each considered value, compared to the base case, all VSI objectives could provide more percentage improvement than those of all VSI constraints due to the minimization of the VSIs for the whole system. However, the increased percentage of the generation costs of VSI objectives are more than those of the VSI constraints because applying VSIs as the constraint can simultaneously satisfy the system economic and security requirements. The higher generation costs represent the additional cost to improve system voltage stability and reduce transmission loss, and the increased generation cost is acceptable when comparing to the decrease of line loss and also the voltage stability improvement for the heavily loaded and line outage contingency situations. So, the operators should evaluate the situation, desired purpose and system size before employing VSIs as part of the constraint or objective function to achieve the most worthy operation. For example, FVSI objective could most improve voltage stability by reaching lowest values of L-index in both systems and both situations, so FVSI objective should be used when the system security is the first priority.

\section{CONCLUSION}

The voltage stability enhancement by employing VSIs as part of the constraint and as the objective function in an OPF problem in the heavily loaded and line outage contingency situations are presented in this work. The OPF problems were solved by using recently proposed SSA. The performance of three line VSIs including Lmn, VCPI and FVSI are compared in terms of loss reduction and voltage stability enhancement. The simulation results express that considering VSIs as the objective function could the best reduce transmission loss and improve system voltage stability compared to those of adding VSIs as part of the constraint and the base case, and adding VSIs as part of the constraint could provide better transmission loss reduction and system voltage stability improvement than those of the base case. It is observed that different VSIs achieved best values in different terms, situations and system sizes for both VSI constraints and VSI objectives. However, the generation costs of VSI objectives are more than those of the VSI constraint and of the base case according to the voltage stability enhancement and loss reduction. These additional costs are acceptable to improve the system security when the system is in the undesirable situations. Hence, by employing VSIs as the objective function or of the constraint, the operators should assess the situation, size and aime of the system, and then select the appropriate VSIs and the objective function. In the future work, other VSIs such as LQP, LVSI or L-index could be taken 
International Journal of Engineering Research and Technology. ISSN 0974-3154, Volume 13, Number 7 (2020), pp. $1635-1648$

(C) International Research Publication House. https://dx.doi.org/10.37624/IJERT/13.7.2020.1635-1648

in consideration to compare the performance in terms of voltage stability improvement and loss reduction.

\section{ACKNOWLEDGMENTS}

This work has received scholarship under the Post-Doctoral Training Program from Khon Kaen University, Thailand.

\section{REFERENCES}

[1] Li, C., Sun, Y., Chen, X., 2007, "Analysis of the blackout in Europe on November 4, 2006," In 2007 International Power Engineering Conference (IPEC 2007); Singapore, pp. 939-944.

[2] Andersson, G., Donalek, P., Farmer, R., Hatziargyriou, N., Kamwa, I., Kundur, P., Martins, N., Paserba, J., Pourbeik, P., Sanchez-Gasca, J., Schulz, R., Stankovic, A., Taylor, C., Vittal, V., 2005, "Causes of the 2003 Major Grid Blackouts in North America and Europe, and Recommended Means to Improve System Dynamic Performance," IEEE Trans. Power Syst., 20(4), pp. 19221928, doi:10.1109/TPWRS.2005.857942.

[3] Ohno, T., Imai, S., 2006, "The 1987 Tokyo blackout," In 2006 IEEE PES Power Systems Conference and Exposition, pp. 314-318.

[4] Berizzi, A., 2004, "The Italian 2003 blackout," In Power Engineering Society General Meeting, 2004. IEEE, Vol. 2, pp. 1673-1679.

[5] Sode-Yome, A., Mithulananthan, N., Lee, K. Y., 2006, "A Maximum Loading Margin Method for Static Voltage Stability in Power Systems," IEEE Trans. Power Syst., 21(2), pp. 799-808, doi:10.1109/TPWRS.2006.873125.

[6] Caizares, C. A., 1995, "On bifurcations, voltage collapse and load modeling," IEEE Trans. Power Syst., 10(1), pp. 512-522, doi:10.1109/59.373978.

[7] Overbye, T. J., DeMarco, C. L., 1991, "Improved Techniques For Power System Voltage Stability Assessment Using Energy Methods," IEEE Trans. Power Syst., 6(4), pp. 1446-1452, doi:10.1109/59.116988.

[8] Flatabo, N., Ognedal, R., Carlsen, T., 1990, "Voltage stability condition in a power transmission system calculated by sensitivity methods," IEEE Trans. Power Syst., 5(4), pp. 1286-1293, doi:10.1109/59.99379.

[9] Kessel, P., Glavitsch, H., 1986, "Estimating the voltage stability of a power system," IEEE Trans. Power Deliv., 1(3), pp. 346-354, doi:10.1109/TPWRD.1986.4308013.

[10] Moghavvemi, M., Omar, F. M., 1998, "Technique for contingency monitoring and voltage collapse prediction," IEE Proc. - Gener. Transm. Distrib., 145(6), pp. 634-640, doi:10.1049/ip-gtd:19982355.

[11] Moghavvemi, M., Faruque, O., 1998, "Real-time contingency evaluation and ranking technique," In IEE Proceedings-Generation, Transmission and Distribution,
Vol. 145, pp. 517-524.

[12] Musirin, I., Rahman, T. K. A., 2002, "Novel fast voltage stability index (FVSI) for voltage stability analysis in power transmission system," In Research and Development, 2002. SCOReD 2002. Student Conference, pp. 265-268.

[13] Mohamed, A., Jasmon, G. B., Yusof, S., 1998, "A static voltage collapse indicator using line stability factors," J. Ind. Technol., 7(1), pp. 73-85.

[14] Moghavvemi, M., Faruque, M. O., 2001, "Technique for assessment of voltage stability in ill-conditioned radial distribution network," IEEE Power Eng. Rev., 21(1), pp. 58-60, doi:10.1109/39.893345.

[15] Ratra, S., Tiwari, R., Niazi, K. R., 2018, "Voltage stability assessment in power systems using line voltage stability index," Comput. Electr. Eng., 70, pp. 199-211, doi:10.1016/j.compeleceng.2017.12.046.

[16] Kanimozhi, R., Selvi, K., 2013, "A novel line stability index for voltage stability analysis and contingency ranking in power system using fuzzy based load flow," J. Electr. Eng. Technol., 8(4), pp. 694-703, doi:10.5370/JEET.2013.8.4.694.

[17] Zabaiou, T., Dessaint, L.-A., Kamwa, I., 2014, "Preventive control approach for voltage stability improvement using voltage stability constrained optimal power flow based on static line voltage stability indices," IET Gener. Transm. Distrib., 8(5), pp. 924-934, doi:10.1049/ietgtd.2013.0724.

[18] Cui, B., Sun, X. A., 2018, "A New Voltage StabilityConstrained Optimal Power-Flow Model: Sufficient Condition, SOCP Representation, and Relaxation," IEEE Trans. Power Syst., 33(5), pp. 5092-5102, doi:10.1109/TPWRS.2018.2801286.

[19] Yang, C. F., Lai, G. G., Lee, C. H., Su, C. T., Chang, G. W., 2012, "Optimal setting of reactive compensation devices with an improved voltage stability index for voltage stability enhancement," Int. J. Electr. Power Energy Syst., 37(1), pp. 50-57, doi:10.1016/j.ijepes.2011.12.003.

[20] Kumar, M., Renuga, P., 2012, "Application of UPFC for enhancement of voltage profile and minimization of losses using Fast Voltage Stability Index (FVSI)," Arch. Electr. Eng., 61(2), pp. 239-250, doi:10.2478/v10171012-0020-4.

[21] Niknam, T., Narimani, M. R., Aghaei, J., AzizipanahAbarghooee, R., 2012, "Improved particle swarm optimisation for multi-objective optimal power flow considering the cost, loss, emission and voltage stability index," IET Gener. Transm. Distrib., 6(6), pp. 515, doi:10.1049/iet-gtd.2011.0851.

[22] Yan, X., Quintana, V. H., 1999, Improving an interiorpoint-based off by dynamic adjustments of step sizes and tolerances," IEEE Trans. Power Syst., 14(2), pp. 709-716, doi:10.1109/59.761902. 
[23] Al-Muhawesh, T. A., Qamber, I. S., 2008, "The established mega watt linear programming-based optimal power flow model applied to the real power 56-bus system in eastern province of Saudi Arabia," Energy, 33(1), pp. 12-21, doi:10.1016/j.energy.2007.08.004.

[24] Burchett, R. C., Happ, H. H., Vierath, D. R., 1984, "Quadratically Convergent Optimal Power Flow," IEEE Trans. Power Appar. Syst., PAS-103(11), pp. 3267-3275, doi:10.1109/TPAS.1984.318568.

[25] Hooshang, H., Luo, G.-X., Semlyen, A., 1989, "Hydrothermal optimal power flow based on a combined linear and nonlinear programming methodology," IEEE Trans. Power Syst., 4(2), pp. 530-537, doi:10.1109/59.193826.

[26] Mohamed, A.-A. A., Mohamed, Y. S., El-Gaafary, A. A. M., Hemeida, A. M., 2017, "Optimal power flow using moth swarm algorithm," Electr. Power Syst. Res., 142, pp. 190-206, doi:10.1016/j.epsr.2016.09.025.

[27] Eberhart, R., Kennedy, J., 1995, "A New Optimizer Using Particle Swarm Theory," Micro Mach. Hum. Sci. 1995. MHS'95., Proc. Sixth Int. Symp., pp. 39-43.

[28] Mirjalili, S., Gandomi, A. H., Mirjalili, S. Z., Saremi, S., Faris, H., Mirjalili, S. M., 2017, "Salp Swarm Algorithm: A bio-inspired optimizer for engineering design problems," Adv. Eng. Softw., 114, pp. 163-191, doi:10.1016/j.advengsoft.2017.07.002.

[29] Khunkitti, S., Siritaratiwat, A., Premrudeepreechacharn, S., Chatthaworn, R., Watson, N., 2018, "A Hybrid DAPSO Optimization Algorithm for Multiobjective Optimal Power Flow Problems," Energies, 11(9), pp. 2270, doi:10.3390/en11092270.

[30] Tolba, M., Rezk, H., Diab, A. A. Z., Al-Dhaifallah, M., 2018, "A novel robust methodology based Salp Swarm Algorithm for allocation and capacity of renewable distributed generators on distribution grids," Energies, 11(10), pp. 2556, doi:10.3390/en11102556.

[31] Abbassi, R., Abbassi, A., Heidari, A. A., Mirjalili, S., 2019, "An efficient salp swarm-inspired algorithm for parameters identification of photovoltaic cell models," Energy Convers. Manag., 179(Octorber 2018), pp. 362372, doi:10.1016/j.enconman.2018.10.069.

[32] El-Fergany, A. A., 2018, Extracting optimal parameters of PEM fuel cells using Salp Swarm Optimizer," Renew. Energy, 119, pp. 641-648, doi:10.1016/j.renene.2017.12.051.

[33] Khunkitti, S., Premrudeepreechacharn, S., Chatthaworn, R., Thasnas, N., Khunkitti, P., Siritaratiwat, A., Watson, N. R., 2019, A comparison of the effectiveness of voltage stability indices in an optimal power flow," IEEJ Trans. Electr. Electron. Eng., 14(4), pp. 534-544, doi:10.1002/tee.22836.

[34] Thasnas, N., Siritaratiwat, A., 2019, Implementation of Static Line Voltage Stability Indices for Improved Static Voltage Stability Margin," J. Electr. Comput. Eng., 2019, doi:10.1155/2019/2609235.

[35] Kumari, M. S., Maheswarapu, S., 2010, Enhanced Genetic Algorithm based computation technique for multiobjective Optimal Power Flow solution," Int. J. Electr. Power Energy Syst., 32(6), pp. 736-742, doi:10.1016/j.ijepes.2010.01.010.

[36] Physiology, I. I., Andrews, S., Andrews, S., 1980, Communication between individuals in salp chains. II. Physiology," In Proceedings of the Royal Society of London. Series B. Biological Sciences, Vol. 210, pp. 559-574.

[37] The University of Washington Electrical Engineering., Power system test case archive, the IEEE 30-bus test system data Available online: https://www2.ee.washington.edu/research/pstca/pf30/pg _tca30bus.htm (accessed on Jan 5, 2020).

[38] The Electrical and Computer Engineering Department, Illinois Institute of Technology, Data, The IEEE 118-bus test system data Available online: http://motor.ece.iit.edu/data/JEAS_IEEE118.doc (accessed on Jan 5, 2020).

[39] Ajjarapu, V., Christy, C., 1992, The continuation power flow: A tool for steady state voltage stability analysis," IEEE Trans. Power Syst., 7(1), pp. 416-423, doi:10.1109/59.141737. 